Editorial: Hospital Metropolitano

ISSN (impreso) 1390-2989 - ISSN (electrónico) 2737-6303

Edición: Vol. 28 No 3 (2020) Julio - Septiembre

DOI: https://doi.org/10.47464/MetroCiencia/vol28/3/2020/25-31

URL: https://revistametrociencia.com.ec/index.php/revista/article/view/83

Pág: 25-31

\title{
Primer reporte ecuatoriano de cirugía bariátrica en ancianos
}

\section{First Ecuadorian report on bariatric surgery in the elderly}

\author{
Andrea Patricia Villarreal Juris ${ }^{1,2}$ (D), Jorge Luis Albán Tigre ${ }^{3,4}$ (D), \\ Hernán Isaí Padilla Paredes ${ }^{5}$ (D), Cristina Joelle Ponce Ontaneda ${ }^{2,6}$ (id \\ Alfredo Daniel Guerrón Cruz ${ }^{2,7}$ (i), William Mesías Puente Galeas ${ }^{3,4}$ (D), \\ Estefany Cristina Garcés Delgado 2,6 iD, Máximo Vicente Torres Guaicha ${ }^{2,4}$ (D), \\ Amílcar Omar Herrera Cevallos 2,4 (D), Glenda Yamira Herrera Cevallos 2,4
}

\author{
Universidad Central del Ecuador, Quito, Ecuador \\ Sociedad Ecuatoriana de Cirugía Bariátrica y Metabólica (SECBAMET); Quito, Ecuador ${ }^{2}$ \\ Pontificia Universidad Católica del Ecuador, Quito, Ecuador ${ }^{3}$ \\ Departamento de Cirugía, Hospital Metropolitano, Quito, Ecuador ${ }^{4}$ \\ Departmento de Cirugía, Clínica Pasteur, Quito, Ecuador ${ }^{5}$ \\ Universidad Internacional del Ecuador, Quito, Ecuador ${ }^{6}$ \\ División de Cirugía Metabólica y de pérdida de peso, Departamento de Cirugía, Duke University, Durham, NC, USA
}

Recibido: 15/06/2020 Aceptado: 10/07/2020 Públicado: 01/09/2020

\section{RESUMEN}

Introducción: la cirugía bariátrica es el tratamiento más eficaz para la obesidad, con beneficios metabólicos adicionales a la pérdida de peso. La evaluación preoperatoria adecuada y planificación quirúrgica son esenciales para su éxito, especialmente en pacientes de edad avanzada. Objetivo: determinar variación de peso, beneficios metabólicos y seguridad de cirugía bariátrica en ancianos, con seguimiento de dos años. Metodología: se incluyeron adultos $\geq 65$ años sometidos a cirugía bariátrica laparoscópica en un hospital terciario privado de referencia en Ecuador, entre 2010 y 2019. Se midió pérdida de peso, mejoría de comorbilidades endocrino-metabólicas y complicaciones. Resultados: se incluyeron 16 pacientes; edad media: 68.5 años (DE: \pm 4.2 ); 12 (75\%) mujeres; $56.25 \%$ se sometió a bypass gástrico; $37.5 \%$ a gastrectomía en manga y $6.25 \%$ a un procedimiento revisional. En dos años, 13 pacientes mostraron un porcentaje de pérdida de peso total (\%TWL) de 31.5\% (DE: $\pm 3.0 \%$ ) y un porcentaje de pérdida de exceso de peso (\% EWL) de $72.9 \%$ (DE: \pm 11.0 ) con bypass gástrico; y de $22.7 \%$ (DE: $\pm 3.2 \%$ ) y $65.6 \%$ (DE: \pm 13.0 ) con gastrectomía en manga, respectivamente. Diabetes mellitus tipo 2 remitió en $80 \%$, hipertensión en $62.5 \%$ y dislipidemia en $60 \%$. El $50 \%$ de complicaciones postoperatorias fueron Grado I según la clasificación Clavien Dindo. Conclusión: este es el primer reporte ecuatoriano sobre cirugía bariátrica en adultos mayores, demostrando su seguridad con baja tasa de complicaciones, su efectividad como tratamiento a corto plazo para la obesidad y sus comorbilidades, y beneficios similares a los presentados en sujetos más jóvenes.

Palabras claves: Cirugía bariátrica, obesidad, anciano, pérdida de peso, complicaciones, seguridad.

\section{ABSTRACT}

Background: bariatric surgery is currently the most effective obesity treatment, with metabolic benefits in addition to weight loss. Adequate preoperative evaluation and surgical planning are essential for success, especially in elderly patients. Objective: determine up to 2-year weight change, metabolic benefits and safety of bariatric surgery in the elderly. Methodology: older adults $\geq 65 y$ who underwent laparoscopic bariatric surgery at a private tertiary referral hospital in Ecuador, between 2010 and 2019 , were included. Weight loss, improvement of endocrine and metabolic comorbidities, and surgical complications were measured up to two years after the procedure. Results: sixteen patients were included. The mean age was 68.5 years (SD: \pm 4.2$) ; 12(75 \%)$ were female. Gastric bypass was performed in $56.25 \%$ of patients; sleeve gastrectomy in $37.5 \%$, and $6.25 \%$ had a revision procedure. Thirteen patients completed a two-year follow-up, where percentage of total weight loss (\%TWL) was $31.5 \%$ (SD: $\pm 3.0 \%$ ), and percentage of excess weight loss (\%EWL) was $72.9 \%$ (SD: \pm 11.0 ) with gastric bypass; and $22.7 \%$ (SD: $\pm 3.2 \%$ ) and $65.6 \%$ (SD: \pm 13.0 ) with sleeve gastrectomy, respectively. Remission for type 2 diabetes mellitus (T2DM) was $80 \%, 62.5 \%$ for hypertension (HT) and $60 \%$ for dyslipidemia. Fifty percent of postoperative complications were Grade I. Conclusion: this is the first report from a tertiary referral hospital in Ecuador about bariatric surgery in the elderly demonstrating its safety with a low complication rate, effectiveness as a short-term treatment for obesity and its comorbidities, and benefits similar to those presented globally in younger subjects.

Keywords: Bariatric surgery, obesity, elderly, weight loss, complications, safety.

Andrea Patricia Villarreal-Juris Jorge Luis Albán-Tigre Hernán Isaí Padilla-Paredes Cristina Joelle Ponce-Ontaneda Alfredo Daniel Guerrón-Cruz Willio Willam Mesias Purte-Galeas Estefany Cristina Garces-Delgado Máximo Vicente Torres-Guaicha Amílcar Omar Herrera-Cevallos Glenda Yamira Herrera-Cevallos

\section{IDs Orcid}

https://orcid.org/0000-0002-0977-3179 https://orcid.org/0000-0002-0456-0561 https://orcid.org/0000-0003-3746-926X https://orcid.org/0000-0001-9019-1856 https://orcid org/0000-0002-4632-1681 htt/// hes:/lorcid.org/0000-0001-6287-5230 hit///rcid.org/0000-0003-0316-0360 htps://orcid.org/0000-0002-4512-5451 https://orcid.org/0000-0001-7490-0210 https://orcid.org/0000-0002-4554-7574

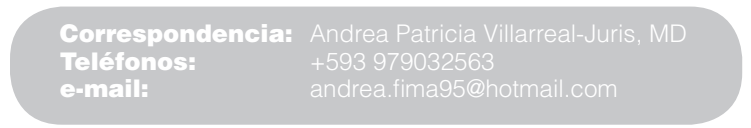




\section{INTRODUCCIÓN}

El envejecimiento de la población, definido como un aumento significativo de la esperanza de vida, se describió por primera vez en los países desarrollados a finales de los años setenta y ochenta. Este fenómeno global se hizo evidente en los países subdesarrollados en las últimas décadas. Según la OMS, para 2050, se espera que el número de adultos mayores se duplique del $11 \%$ al $22 \%{ }^{1,2}$.

Junto con el aumento de la esperanza de vida, se produce un aumento posterior de las comorbilidades, especialmente las enfermedades crónicas no transmisibles, incluida la obesidad. En Estados Unidos (EE. UU.), el $42.5 \%$ de las mujeres y el $38.1 \%$ de los hombres entre 60 y 69 años son obesos. En Ecuador, una encuesta nacional en 2010 informó que el 59\% de los adultos mayores tenían sobrepeso ${ }^{3,4}$. La Encuesta Nacional de Salud y Nutrición de Ecuador de 2012 (ENSANUT-ECU) informó que 6 de cada diez ecuatorianos adultos sufren de sobrepeso u obesidad en el 70\% del territorio del país ${ }^{5,6}$. En 2016, un informe de la OMS, que incluía a los ancianos, indicó que el $14.9 \%$ de los hombres y el $24.7 \%$ de las mujeres mayores de 18 años eran obesos (IMC> $\left.30 \mathrm{~kg} / \mathrm{m}^{2}\right)^{7,8}$. Varios factores pueden explicar la mayor prevalencia de la obesidad en las mujeres, como la inseguridad alimentaria que conduce a una mayor ingesta de alimentos ricos en calorías o pobres en nutrientes, gradientes de ingresos y educación, junto con un aumento significativo de la esperanza de vida ${ }^{9}$. En Ecuador, la prevalencia de sobrepeso u obesidad en mujeres adultas es 5.5 puntos porcentuales mayor que en los hombres $(65.5 \% \text { vs } 60 \%)^{5}$.

Para la obesidad, tradicionalmente se ha propuesto la dieta y ejercicio como primera línea de tratamiento. Sin embargo, en los ancianos, los resultados a mediano y largo plazo con este enfoque son discutibles debido a la fragilidad y dependencia de este grupo etario, lo que podría dificultar mantener cambios en su comportamiento alimentario y actividad física ${ }^{10-12}$.

En 1991, los Institutos Nacionales de Salud (NIH) de EE. UU. establecieron la edad ideal para un procedimiento bariátrico entre 18 y 60 años. Desde entonces, este estándar ha sufrido varias modificaciones y, en la actualidad, no existe un límite superior para la edad. Siendo las indicaciones para cirugía bariátrica en los ancianos las mismas que para sujetos más jóvenes ${ }^{14}$ : índice de masa corporal (IMC) igual o superior a $40 \mathrm{~kg} / \mathrm{m}^{2}$ o igual o superior a $35 \mathrm{~kg} / \mathrm{m}^{2}$ asociado a comorbilidades relacionadas con la obesidad $^{15}$.

En EE. UU., los procedimientos bariátricos en pacientes ancianos han aumentado del $2.7 \%$ en 1999-2005 al 10.1\% en 2009-20136. En este último período, la morbilidad en ancianos fue $1.33 \%$ y la mortalidad $0.11 \%$, frente a $0.70 \%$ en 1999-2005. Asimismo, la morbilidad en adultos más jóvenes ascendió a $0.73 \%$ y la mortalidad a $0.05 \%$, frente a $0.30 \%$ en $1999-2005$. Estos cambios probablemente están relacionados con la evolución de las técnicas laparoscópicas, el aumento del volumen quirúrgico y los programas de acreditación de "centros de excelencia" iniciados en 2004 y 2005 por la Sociedad Estadounidense de Cirugía Bariátrica y Metabólica (ASMBS) y el Colegio Estadounidense de Cirujanos (ACS), respectivamente ${ }^{16}$.

En este estudio, se describen los resultados en cuanto a control de peso, mejoría y remisión de comorbilidades endocrino-metabólicas, complicaciones y seguridad general de los procedimientos bariátricos en adultos mayores ecuatorianos con un seguimiento de dos años.

\section{MATERIALES Y MÉTODOS}

\section{Selección de pacientes}

Se realizó un estudio observacional retrospectivo, incluyendo adultos de 65 años o más sometidos a cirugía bariátrica laparoscópica en el Hospital Metropolitano, Quito-Ecuador, desde 2010 hasta 2019. Se recolectaron datos a partir de historias clínicas electrónicas (HCE) almacenadas en los servidores del Hospital Metropolitano con autorización previa del Comité de Investigación del Hospital. Se excluyeron los pacientes menores de 65 años o aquellos con datos incompletos en su expediente.

El procedimiento se indicó en base a un IMC igual o superior a $40 \mathrm{~kg} / \mathrm{m}^{2}$, o igual o superior a $35 \mathrm{~kg} / \mathrm{m}^{2}$ con comorbilidades asociadas a síndrome metabólico. Tras la evaluación por parte de un equipo multidisciplinario, se seleccionó el tipo de cirugía en función de los objetivos terapéuticos de cada paciente, y se adaptó a sus factores de riesgo específicos.

\section{Técnica quirúrgica}

Todos los procedimientos se completaron por vía laparoscópica. Brevemente, se introdujo el primer trócar bajo visión directa (sin bisturí) y se estableció el neumoperitoneo. Se colocaron cinco puertos en la parte superior del abdomen. La gastrectomía en manga se completó mediante disparo secuencial de una grapadora laparoscópica sobre un bougie 36 $\mathrm{Fr}$, y el bypass gástrico se completó con una engrapadora lineal para la gastroyeyunostomía y yeyunoyeyunostomía sobre un bougie $32 \mathrm{Fr}$, con asas alimentarias y biliopancreáticas de $100 \mathrm{~cm}$. Todos los defectos comunes se cerraron con sutura absorbible trenzada. Los defectos mesentéricos y de Petersen se cerraron con sutura no absorbible ${ }^{17,18}$. 


\section{Resultados de interés}

Las variables clínicas de interés se extrajeron manualmente de las HCE. Los resultados del procedimiento bariátrico reportaron mediante el porcentaje de pérdida de peso total (\%TWL) dado por la fórmula [(peso inicial - peso actual) / (peso inicial)] $\times$ 100 ${ }^{19-21}$, el porcentaje de pérdida de IMC dado por la fórmula (IMC inicial - IMC final / IMC inicial) $x$ $100^{22}$, y el porcentaje de pérdida de exceso de peso (\%EWL), dado por la fórmula [(peso inicial - peso actual) / (peso inicial - peso ideal)] x 100, utilizando un IMC ideal de $25 \mathrm{~kg} / \mathrm{m}^{2}$ 23,24. Estos parámetros, al igual que la mejoría y remisión de comorbilidades, se evaluaron al año y dos años posquirúrgicos, con un período de ventana de 3 meses para la recolección de datos.

La medición objetiva de las complicaciones posoperatorias se realizó a través de la clasificación de Clavien Dindo ${ }^{25-27}$ basada en los datos de la historia clínica (Anexo 1).

Anexo 1. Clasificación según Clavien Dindo 25,26

\begin{tabular}{cl}
\multicolumn{2}{c}{ Complicaciones según Clavien Dindo } \\
\hline Grado & No Complicaciones \\
\hline C & Cualquier desviación en el postoperatorio \\
& que no requiera reintegración a cielo abier- \\
to o endoscópica, uso de soluciones, anti- \\
heméticos, antipiréticos, analgésicos, fisio- \\
terapia \\
II
\end{tabular}

Con respecto a la progresión de comorbilidades, una mejoría se definió como una disminución en la dosis o el número de medicamentos necesarios para mantener una $\mathrm{HbA} 1 \mathrm{c}$ por debajo de $6.0 \%{ }^{28}$, una presión arterial por debajo de 130/80 mmHg $\mathrm{mm}^{29,30}$ y un colesterol <200 mg/dl con triglicéridos <150 mg/dl24,31, para diabetes mellitus tipo 2 , hipertensión y trastornos del metabolismo de los lípidos, respectivamente. La remisión se definió como ausencia total de medicación para lograr los objetivos mencionados anteriormente. Para trastornos osteoarticulares, la mejoría se definió como la reducción del dolor o los fármacos necesarios para controlar el dolor, mientras que la remisión significó ausencia de dolor sin uso de medicación analgésica ${ }^{32}$.

\section{Análisis estadístico}

Se realizó un análisis descriptivo para datos demográficos (edad, sexo, altura), IMC y cambio de peso (peso pre y posquirúrgico, \%TWL, \%EWL), tipo de cirugía, tiempo quirúrgico, estancia hospitalaria, prevalencia y evolución de comorbilidades relacionadas a la obesidad; así como complicaciones según Clavien Dindo. Las variables continuas se expresaron como media \pm desviación estándar, mientras que las variables categóricas se reportaron como recuento y porcentaje de columna. La prueba T de Student se realizó con un intervalo de confianza del 95\% para \%TWL, \%EWL y porcentaje de pérdida de IMC. El análisis estadístico se realizó utilizando la herramienta estadística JASP 0.11.1.0 respaldada por la Universidad de Amsterdam y $p<0.05$ se consideró estadísticamente significativo.

\section{RESULTADOS}

\section{Población del estudio}

En el Hospital Metropolitano, 308 pacientes fueron sometidos a cirugía bariátrica entre 2010 y 2019, 292 menores de 65 años. Dieciséis pacientes cumplieron los criterios de inclusión para este estudio; todos completaron un seguimiento de 1 año, pero solo 13 completaron un seguimiento de dos años. Doce $(75 \%)$ fueron mujeres y 4 (20\%) fueron hombres, con una edad media de 69.2 años (DE: \pm 4.5 años) y 66.5 años (DE: \pm 1.9 años); e IMC medio de $43.0 \mathrm{~kg} / \mathrm{m} 2$ (DE: \pm 4.7 ) y $38.9 \mathrm{~kg} / \mathrm{m} 2$ (DE: \pm 3.3 ), respectivamente. Junto con lo anterior, en la Tabla 1 se describen otras características demográficas como el peso y la talla medios, el tipo de obesidad y la prevalencia de comorbilidades.

Tabla 1. Datos demográficos

\begin{tabular}{cccc} 
& Total & Masculino & Femenino \\
\hline Pacientes & $16(100 \%)$ & $4(25 \%)$ & $12(75 \%)$ \\
Edad media (años) & $68.5( \pm 4.2)$ & $66.5( \pm 1.9)$ & $69.2( \pm 4.5)$ \\
Peso $(\mathrm{kg})$ & $112.4( \pm 13.4)$ & $107.6( \pm 7.3)$ & $114.0( \pm 14.7)$ \\
Talla $(\mathrm{m})$ & $1.64( \pm 0.1)$ & $1.67( \pm 0.1)$ & $1.63( \pm 0.1)$ \\
IMC $\left(\mathrm{kg} / \mathrm{m}^{2}\right)$ & $41.9( \pm 4.6)$ & $38.9( \pm 3.3)$ & $43.0( \pm 4.7)$ \\
DMT2 & $8(50 \%)$ & $1(12.5 \%)$ & $7(87.5 \%)$ \\
HT & $8(50 \%)$ & $3(37.5 \%)$ & $5(62.5 \%)$ \\
Dislipidemia & $5(31.3 \%)$ & $1(20 \%)$ & $4(80 \%)$ \\
Trastornos OA & $4(25 \%)$ & $1(25 \%)$ & $3(75 \%)$ \\
\hline
\end{tabular}

* Los valores se presentan como No. (\%) o media (DE) según corresponda.

IMC: índice de masa corporal; DMT2, diabetes mellitus tipo 2; HT, hipertensión; OA, osteoarticular.

\section{Procedimiento quirúrgico y seguridad}

La mayoría de los participantes se sometieron a un bypass gástrico; uno de ellos fue convertido a técnica abierta debido a adherencias abdominales seve- 
ras secundarias a cesáreas previas. Un paciente se sometió a una cirugía revisional de gastrectomía en manga a bypass gástrico debido a enfermedad por reflujo gastroesofágico (ERGE) con esofagitis (Tabla 2).

Tabla 2. Tipo de cirugía según el sexo

\begin{tabular}{ccccc} 
& $\begin{array}{c}\text { Bypass } \\
\text { gástrico }\end{array}$ & $\begin{array}{c}\text { Gastrectomía } \\
\text { en manga }\end{array}$ & $\begin{array}{c}\text { Conversión } \\
\text { abierta }\end{array}$ & $\begin{array}{c}\text { Bypass } \\
\text { revisional }\end{array}$ \\
\hline Masculino & $2(12.5 \%)$ & $2(12.5 \%)$ & $0(0 \%)$ & $0(0 \%)$ \\
Femenino & $6(37.5 \%)$ & $4(25 \%)$ & $1(6.25 \%)$ & $1(6.25 \%)$ \\
Total & $8(50 \%)$ & $6(37.5 \%)$ & $1(6.25 \%)$ & $1(6.25 \%)$ \\
\hline * Los valores se presentan como No. (\%). & & \\
\hline
\end{tabular}

El tiempo quirúrgico medio global fue de $132.1 \mathrm{mi}-$ nutos (DE: \pm 81.6 minutos). Para bypass gástrico, el tiempo quirúrgico medio fue 113 minutos (DE: \pm 46.7 minutos), prolongado hasta 330 minutos en cirugía revisional y 305 minutos cuando se convirtió a técnica abierta. Para la gastrectomía en manga, fue 95.8 minutos (DE: \pm 30.9 minutos).

En cuanto a las complicaciones tempranas según la clasificación de Clavien Dindo, el 25\% de los pacientes no presentó ninguna complicación, el 50\% presentó complicaciones Grado I, el 18.8\% Grado II y el $6.2 \%$ Grado III; no hubo complicaciones graves.

La estancia hospitalaria media fue 49.8 horas (DE: \pm 27 horas). Durante el seguimiento, ningún participante fue readmitido.

\section{Variación de peso}

El IMC disminuyó a $32.4 \mathrm{~kg} / \mathrm{m}^{2}$ (DE: \pm 3.5) al año de seguimiento y a $30.3 \mathrm{~kg} / \mathrm{m}^{2}$ (DE: \pm 3.0 ) a los dos años para los trece pacientes que completaron el seguimiento a este tiempo, con un porcentaje de pérdida de IMC de $9.6 \%$ (DE: $\pm 3.0 \%$; 5.9-15.3) y $11.6 \%$ (DE: $\pm 3.2 \% ; 7.1-16.6)$, respectivamente. El porcentaje de pérdida de peso total (\%TWL) fue $22.7 \%$ (DE: \pm $5.8 \%$; 14.6-33.2) en el primer año y $27.4 \%$ (DE: \pm $5.5 \%$; 18.7-35.5) en el segundo año. El porcentaje de pérdida de exceso de peso (\%EWL) fue $57.4 \%$ (DE: $\pm 13.2 \%$; 30.6-85.2) en el primer año y $69.5 \%$ (DE: \pm $12.1 \% ; 47.7-91.2)$ en el segundo año.

La prueba T de Student mostró que el \%TWL, \%EWL y el porcentaje de pérdida de IMC fueron todos estadísticamente significativos a uno y dos años de seguimiento, con un gran efecto (Tabla 3).
Tabla 3. Prueba $T$ de Student

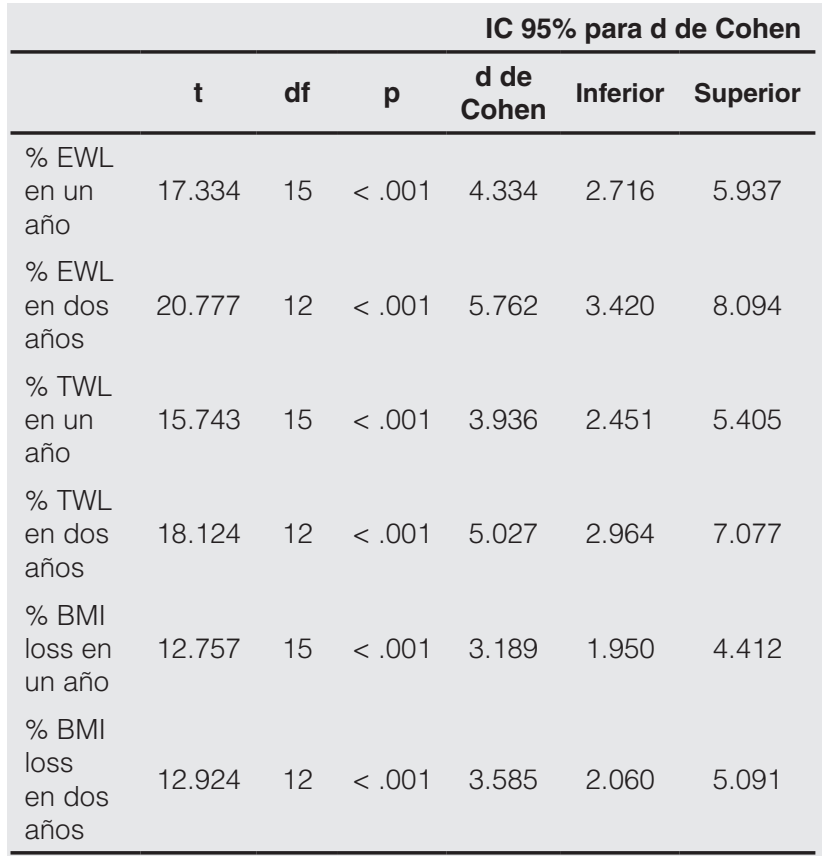

* Para la prueba T de Student, el tamaño del efecto viene dado por la d de Cohen.

EWL: pérdida de exceso de peso; TWL: pérdida de peso total; IMC indice de masa corporal.

El \%TWL y el \%EWL fueron mayores con el bypass gástrico en comparación con la gastrectomía en manga tanto en el primer como en el segundo año después del procedimiento. Estos resultados, junto con la evolución del IMC según el tipo de cirugía, se describen en la Tabla 4 y la Figura 1.

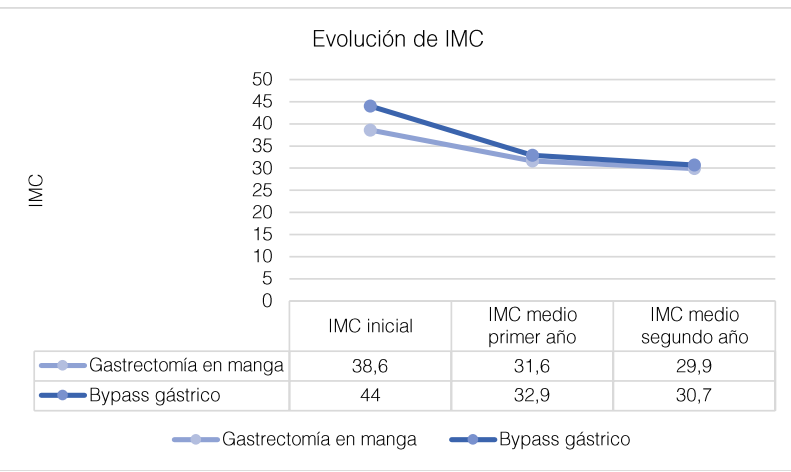

Figura 1. Evolución de IMC

IMC: índice de masa corporal $\left(\mathrm{kg} / \mathrm{m}^{2}\right)$ 
Tabla 4. Evolución de IMC según el tipo de cirugía

\begin{tabular}{|c|c|c|c|c|c|c|c|c|c|}
\hline \multirow{2}{*}{$\begin{array}{l}\text { Tipo de } \\
\text { cirugía }\end{array}$} & \multirow{2}{*}{$\begin{array}{c}\text { IMC } \\
\text { inicial } \\
\left(\mathrm{kg} / \mathrm{m}^{2}\right)\end{array}$} & \multicolumn{2}{|c|}{ IMC primer año } & \multicolumn{2}{|c|}{ IMC segundo año } & \multirow{2}{*}{$\begin{array}{c}\text { \%TWL } \\
\text { primer } \\
\text { año }\end{array}$} & \multirow{2}{*}{$\begin{array}{l}\text { \%TWL } \\
\text { Segundo } \\
\text { año }\end{array}$} & \multirow{2}{*}{$\begin{array}{c}\text { \%EWL } \\
\text { primer } \\
\text { año }\end{array}$} & \multirow{2}{*}{$\begin{array}{c}\text { \%EWL } \\
\text { Segundo } \\
\text { año }\end{array}$} \\
\hline & & $\begin{array}{c}\text { Media } \\
\left(\mathrm{kg} / \mathrm{m}^{2}\right)\end{array}$ & $\begin{array}{c}\% \\
\text { Reducción }\end{array}$ & $\begin{array}{c}\text { Media } \\
\left(\mathrm{kg} / \mathrm{m}^{2}\right)\end{array}$ & $\begin{array}{c}\% \\
\text { Reducción }\end{array}$ & & & & \\
\hline $\begin{array}{c}\text { Gastrectomía } \\
\text { en manga }\end{array}$ & $\begin{array}{c}38.6 \\
( \pm 2.3)\end{array}$ & $\begin{array}{c}31.6 \\
( \pm 1.4)\end{array}$ & $\begin{array}{l}7.0 \% \\
( \pm 1.1)\end{array}$ & $\begin{array}{l}29.9 \\
( \pm 2.5)\end{array}$ & $\begin{array}{l}8.7 \% \\
( \pm 1.1)\end{array}$ & $\begin{array}{l}18.1 \% \\
( \pm 2.0)\end{array}$ & $\begin{array}{l}22.7 \% \\
( \pm 3.2)\end{array}$ & $\begin{array}{l}51.8 \% \\
( \pm 3.8)\end{array}$ & $\begin{array}{l}65.6 \% \\
( \pm 13.0)\end{array}$ \\
\hline $\begin{array}{l}\text { Bypass gás- } \\
\text { trico }\end{array}$ & $\begin{array}{c}44.0 \\
( \pm 4.6)\end{array}$ & $\begin{array}{c}32.9 \\
( \pm 4.4)\end{array}$ & $\begin{array}{l}11.2 \% \\
( \pm 2.7)\end{array}$ & $\begin{array}{l}30.7 \\
( \pm 3.3)\end{array}$ & $\begin{array}{l}14.2 \% \\
( \pm 2.0)\end{array}$ & $\begin{array}{l}25.4 \% \\
( \pm 5.6)\end{array}$ & $\begin{array}{l}31.5 \% \\
( \pm 3.0)\end{array}$ & $\begin{array}{c}60.7 \% \\
( \pm 15.8)\end{array}$ & $\begin{array}{l}72.9 \% \\
( \pm 11.0)\end{array}$ \\
\hline
\end{tabular}

\section{Evolución de comorbilidades}

El control de comorbilidades se realizó mediante la evaluación de la presencia de la enfermedad antes de la cirugía, su mejoría y remisión al primer y segundo año de seguimiento. Los porcentajes de estos resultados varían de una comorbilidad a otra, siendo la diabetes mellitus la enfermedad con mayores tasas de mejoría y remisión al año y dos años; seguida de dislipidemia (Tabla 5).

Tabla 5. Evolución de comorbilidades

\begin{tabular}{|c|c|c|c|c|}
\hline \multirow{2}{*}{ Patología } & \multicolumn{2}{|c|}{ Primer año } & \multicolumn{2}{|c|}{ Segundo año } \\
\hline & Mejoría & Remisión & Mejoría & Remisión \\
\hline \multirow[b]{2}{*}{ DMT2 } & $7 / 8$ & $5 / 8$ & $5 / 5$ & $4 / 5$ \\
\hline & $87.5 \%$ & $625 \%$ & $100 \%$ & $80 \%$ \\
\hline \multirow{3}{*}{$\mathrm{HT}$} & $5 / 8$ & $3 / 8$ & $7 / 8$ & $5 / 8$ \\
\hline & & & & \\
\hline & $62.5 \%$ & $37.5 \%$ & $87.5 \%$ & $62.5 \%$ \\
\hline \multirow{3}{*}{ Dislipidemia } & $3 / 5$ & $2 / 5$ & $4 / 5$ & $3 / 5$ \\
\hline & & & & \\
\hline & $60 \%$ & $40 \%$ & $80 \%$ & $60 \%$ \\
\hline \multirow{2}{*}{$\begin{array}{c}\text { Trastornos } \\
\text { Osteoarticulares }\end{array}$} & $4 / 4$ & $2 / 4$ & $1 / 4$ & $0 / 4$ \\
\hline & $100 \%$ & $50 \%$ & $25 \%$ & $0 \%$ \\
\hline $\begin{array}{l}{ }^{*} \text { Los valores se pr } \\
\text { que lograron el se } \\
\text { DMT2: diabetes }\end{array}$ & $\begin{array}{l}\text { entan con } \\
\text { miento } \\
\text { tus tipo } 2\end{array}$ & $\begin{array}{l}\text { No. }(\%) d \\
\text { HT: hiperte }\end{array}$ & $\begin{array}{l}\text { el núme } \\
\text { on. }\end{array}$ & pacientes \\
\hline
\end{tabular}

\section{DIscusıón}

Este es el primer reporte que expone los resultados de la cirugía bariátrica en adultos mayores en una población ecuatoriana. Los resultados sugieren que la cirugía bariátrica es un tratamiento eficaz para la obesidad y sus comorbilidades en este grupo etario, con una baja tasa de complicaciones, demostrando resultados similares a los estudios realizados en otros países ${ }^{33}$. Los pacientes adultos mayores que se sometieron a bypass gástrico o gastrecto- mía en manga mostraron una reducción de peso estadísticamente significativa, obteniendo valores de \%TWL y \%EWL concordantes con aquellos descritos en otros estudios: $\geq 20 \%$ para el primero $^{19}$, y $\geq 50 \%$ para el segundo, de acuerdo con los criterios de Reinhold ${ }^{21,23}$, tanto a uno como a dos años de seguimiento. Las complicaciones descritas en este análisis son en su mayoría leves, de manera similar a aquellas reportadas en estudios de adultos jóvenes ${ }^{14,34}$, demostrando la seguridad de la cirugía bariátrica en adultos mayores, así como lo han determinado otros artículos publicados ${ }^{35,36}$; apoyando así la afirmación de que una edad avanzada por sí sola no debe considerarse una contraindicación para el tratamiento quirúrgico de la obesidad. El sexo femenino predominó en este estudio y el IMC inicial fue mayor en este grupo. La edad, así como la altura, tuvieron poca variabilidad entre los sexos. Es importante reconocer que los ecuatorianos tienden a tener una estructura corporal más pequeña y extremidades más cortas en comparación con la población mundial, y el cálculo del IMC puede no estimar con precisión el exceso de grasa corporal ${ }^{37}$.

La mayoría de las intervenciones quirúrgicas fueron bypass gástrico, al igual que en varios estudios ${ }^{38}$.

De manera semejante a otros estudios realizados en adultos $^{39}$, la mayoría de las comorbilidades lograron mejoría y remisión durante el período de seguimiento. Cabe señalar que los pacientes que padecían trastornos osteoarticulares mostraron mejoría después de un año de seguimiento, posiblemente debido a la reducción de la carga de peso en las articulaciones. Sin embargo, ninguno de los pacientes mantuvo la remisión un año después, probablemente debido al envejecimiento intrínseco de los individuos y al curso crónico degenerativo irreversible de la enfermedad.

A pesar de estos resultados favorables, existen varias limitaciones para el presente estudio, comenzando por su diseño retrospectivo. Además, este fue llevado a cabo en un solo centro de referencia terciario con cirujanos bariátricos expertos. Si bien se demostró la viabilidad y seguridad de la cirugía bariátrica en los adultos mayores a corto plazo, la 
reproducibilidad del estudio en diferentes entornos aún no se ha determinado.

No se informaron comorbilidades no asociadas con la obesidad o el síndrome metabólico y pueden representar un efecto de confusión sobre los resultados obtenidos. Además, el hecho de que tres pacientes no completaran un seguimiento de dos años podría modificar los resultados a este tiempo y puede considerarse una limitación.

El tamaño de la muestra limitó la potencia de algunos análisis, pero, incluso con un tamaño de muestra pequeño, los resultados presentados aún pueden considerarse una contribución significativa ya que no se han publicado muchos artículos sobre cirugía bariátrica en adultos mayores en América Latina y ninguno en Ecuador. Sin embargo, se necesita más investigación para corroborar estos hallazgos con un mayor tamaño de muestra y un seguimiento a largo plazo en el futuro. Además, debe tenerse en cuenta el hecho de que Ecuador está compuesto por cuatro regiones distintas que incluyen la Cordillera de los Andes, Amazonía, las costas del Pacífico y las Islas Galápagos, pudiendo existir variabilidad evidente de los resultados en adultos mayores debido a la naturaleza multicultural de su población ${ }^{40}$.

\section{CONCLUSIÓN}

La cirugía bariátrica es una opción adecuada para el tratamiento a corto plazo de la obesidad y sus comorbilidades en el adulto mayor. Parece ser un procedimiento seguro con baja tasa de complicaciones y beneficios similares a los presentados en la población mundial. Se necesitan más estudios para evaluar los resultados a largo plazo de este tipo de cirugía, con el fin de actualizar las guías de tratamiento y estandarizar las recomendaciones de indicaciones y protocolo quirúrgico en la cirugía bariátrica como tratamiento de la obesidad en este grupo etario.

\section{Conflictos de interés}

Un autor ha recibido honorarios como consultor para Levita y como orador para Gore y Medtronic. Los demás autores declaran no tener ningún conflicto de intereses. Los autores declaran la originalidad del trabajo; no ha sido publicado en ningún medio antes.

\section{Aspectos Éticos}

Los autores declaran que los procedimientos seguidos en este estudio fueron exclusivamente analizados retrospectivamente, y de acuerdo con los estándares éticos del comité institucional de investigación y con la declaración de Helsinki de 1964 y sus posteriores modificaciones o estándares éticos comparables. Para este tipo de estudio no se requiere consentimiento formal.

\section{Financiamiento}

Los autores no recibieron apoyo económico para la investigación, autoría o publicación de este artículo.

\section{Contribución}

Andrea Patricia Villarreal-Juris: contribución sustancial al diseño de la investigación; análisis e interpretación de datos; redacción y revisión crítica del documento; aprobación de la versión presentada.

Jorge Luis Albán-Tigre: contribución sustancial al diseño de la investigación; análisis e interpretación de datos; revisión crítica del documento; aprobación de la versión presentada. Hernán Isaí Padilla-Paredes: recolección y análisis de datos; revisión crítica del documento; aprobación de la versión presentada.

Cristina Joelle Ponce-Ontaneda: revisión crítica del documento; aprobación de la versión presentada.

Alfredo Daniel Guerrón-Cruz: revisión crítica del documento; aprobación de la versión presentada.

William Mesías Puente-Galeas: análisis de datos; revisión crítica del documento; aprobación de la versión presentada.

Estefany Cristina Garcés-Delgado: recolección de datos; aprobación de la versión presentada. Máximo Vicente Torres-Guaicha: contribución sustancial al diseño de la investigación; aprobación de la versión presentada.

Amilcar Omar Herrera-Cevallos: contribución sustancial al diseño de la investigación; aprobación de la versión presentada.

Glenda Yamira Herrera-Cevallos: contribución sustancial al diseño de la investigación; revisión crítica del documento; aprobación de la versión presentada.

\section{REFERENCIAS BIBLIOGRÁFICAS}

1. Cazzo E, Gestic MA, Utrini MP, et al. Bariatric surgery in the elderly: A narrative review. Rev Assoc Med Bras. 2017;63(9):787-792. doi:10.1590/1806-9282.63.09.787 PMID: 29239469

2. Bowers B. Caregiving in an Aging World: Quality of Care and Quality of Life. In: 29th International Nursing Research Congress Melbourne-Australia. ; 2018.

3. Diario La Hora. En Ecuador el 59\% de adultos sufren de obesidad País : La Hora Noticias de Ecuador, sus provincias y el mundo. https:// lahora.com.ec/noticia/1101051854/en-ecuador-el-59_-de-adultos-sufren-de-obesidad. Published 2010. Accessed June 24, 2020.

4. INEC. Encuesta de Salud, Bienestar del Adulto Mayor. https://www. ecuadorencifras.gob.ec/encuesta-de-salud-bienestar-del-adulto-mayor/. Accessed June 24, 2020.

5. Freire WB, Ramírez MJ, Belmont $\mathbf{P}$, et al. Encuesta Nacional de Salud y Nutrición. ENSANUT-ECU 2011-2013. Vol 1. Ministerio de Salud Pública; 2013.

6. Freire WB, Silva-Jaramillo KM, Ramírez-Luzuriaga MJ, Belmont P, Waters WF. The double burden of undernutrition and excess body weight in Ecuador. Am J Clin Nutr. 2014;100(6):1636S-43S. doi:10.3945/ajcn.114.083766 PMID: 25411306 
7. Zamboni M, Mazzali G. Obesity in the elderly: An emerging health issue. Int J Obes. 2012;36(9):1151-1152. doi:10.1038/ijo.2012.120 PMID: 22964828

8. WHO. Overweight and obesity. https://www.who.int/gho/ncd/risk_factors/overweight_obesity/obesity_adults/en/. Published 2018. Accessed June 24, 2020

9. Hruby A, Hu FB. The Epidemiology of Obesity: A Big Picture. Pharmacoeconomics. 2015;33(7):673-689. doi:10.1007/s40273-014-0243-x

10. WHO. Obesity: preventing and managing the global epidemic. https:// www.who.int/nutrition/publications/obesity/WHO_TRS_894/en/. Published 2015. Accessed June 24, 2020

11. Schauer PR, Bhatt DL, Kirwan JP, et al. Bariatric surgery versus intensive medical therapy for diabetes - 5-year outcomes. N Engl Med. 2017;376(7):641-651. doi:10.1056/NEJMoa1600869. PMID: 28199805

12. Pajecki D, Santo MA, Joaquim HDG, et al. Bariatric Surgery in the Elderly: Results of a mean follow-up of five years. Arq Bras Cir Dig. 2015;28:15-18. doi:10.1590/S0102-6720201500S100006

13. Rigamonti AE, De Col A, Tamini S, et al. Multidisciplinary integrated metabolic rehabilitation in elderly obese patients: Effects on cardiovascular risk factors, fatigue and muscle performance. Nutrients. 2019;11(6). doi:10.3390/nu11061240 PMID: 31159183

14. Albán-Tigre JL, Villarreal-Juris AP, Puente-Galeas WM, Padilla-Paredes $\mathbf{H}$, Albán-Tigre CA, Yamira-Herrera G. Seguridad y Resultados de Cirugía Bariátrica en Adolescentes: Seguimiento a dos años. Rev la Soc Peru Cirugía Endoscópica. 2019;1(2):52-56. ISSN: 2664-2417

15. Susmallian S, Raziel A, Barnea R, Paran H. Bariatric surgery in older adults: Should there be an age limit? Medicine (Baltimore) 2019;98(3):e13824. doi:10.1097/MD.0000000000013824 PMID: 30653091

16. Gebhart A, Young MT, Nguyen NT. Bariatric surgery in the elderly: 2009-2013. Surg Obes Relat Dis. 2015;11(2):393-398. doi:10.1016/j. soard.2014.04.014 PMID: 25130515

17. Nguyen NT, De Maria EJ, Ikramuddin S, Hutter MM. The SAGES Manual: A Practical Guide to Bariatric Surgery. Springer New York; 2008. doi:10.1007/978-0-387-69171-8

18. Takahashi H, Allemang MT, Strong AT, et al. Completion gas trectomy with esophagojejunostomy for management of complications of benign foregut surgery. J Laparoendosc Adv Surg Tech A 2018;28(8):983-989. doi:10.1089/lap.2017.0540 PMID: 29493349

19. Sabench Pereferrer F, Molina López A, Vives Espelta M, et al. Weight Loss Analysis According to Different Formulas after Sleeve Gastrectomy With or Without Antral Preservation: a Randomised Study Obes Surg. 2017;27(5):1254-1260. doi:10.1007/s11695-016-2454-Z PMID: 27995517

20. Corcelles R, Boules M, Froylich D, et al. Total Weight Loss as the Outcome Measure of Choice After Roux-en-Y Gastric Bypass. Obes Surg. 2016;26(8). doi:10.1007/S11695-015-2022-Y PMID: 26803753

21. Grover BT, Morell MC, Kothari SN, Borgert AJ, Kallies KJ, Bake MT. Defining Weight Loss After Bariatric Surgery: a Call for Standardization. Obes Surg. 2019;29(11):3493-3499. doi:10.1007/s11695-01904022-z PMID: 31256357

22. Larrad Á, Sánchez-Cabezudo C. Indicadores de calidad en cirugía bariátrica y criterios de éxito a largo plazo. Cir Esp (Ed impr). 2004:301-304. ID: ibc-31922

23. Dimeglio C, Becouarn G, Topart P, Bodin R, Buisson JC, Ritz P. Weight loss trajectories after bariatric surgery for obesity: Mathematical model and proof-of-concept study. J Med Internet Res. 2020;22(3). doi:10.2196/13672 PMID: 32149710

24. Brethauer SA, Kim J, El Chaar M, et al. Standardized outcomes reporting in metabolic and bariatric surgery. Surg Obes Relat Dis. 2015;11(3):489-506. doi:10.1016/j.soard.2015.02.003 PMID: 26093765
25. Dindo D, Demartines N, Clavien PA. Classification of surgical complications: A new proposal with evaluation in a cohort of 6336 patients and results of a survey. Ann Surg. 2004;240(2):205-213. doi:10.1097/01.sla.0000133083.54934.ae PMID: 15273542

26. Clavien PA, Barkun J, De Oliveira ML, et al. The clavien-dindo classification of surgical complications: Five-year experience. Ann Surg 2009;250(2):187-196. doi:10.1097/SLA.0b013e3181b13ca2 PMID: 19638912

27. Welsh LK, Luhrs AR, Davalos G, et al. Racial Disparities in Bariatric Surgery Complications and Mortality Using the MBSAQIP Data Registry. Obes Surg. 2020;30(8). doi:10.1007/s11695-020-04657-3 PMID: 32388704

28. Ikramuddin S, Korner J, Lee WJ, et al. RouX-en-Y gastric bypass vs intensive medical management for the control of type 2 diabetes, hypertension, and hyperlipidemia: The diabetes surgery study randomized clinical trial. JAMA - J Am Med Assoc. 2013:309(21):22402249. doi:10.1001/jama.2013.5835 PMID: 23736733

29. Arnett DK, Blumenthal RS, Albert MA, et al. 2019 ACC/AHA Guideline on the Primary Prevention of Cardiovascular Disease: A Report of the American College of Cardiology/American Heart Association Task Force on Clinical Practice Guidelines. Circulation. 2019;140(11):e596-e646. doi:10.1161/CIR.0000000000000678 PMID: 30879355

30. Beamish AJ, Olbers T, Kelly AS, Inge TH. Cardiovascular effects of bariatric surgery. Nat Rev Cardiol. 2016:13(12):730-743. doi:10.1038/ nrcardio.2016.162 PMID: 27762312

31. Instituto Mexicano del Seguro Social. Diagnóstico y tratamiento de dislipidemias. In: Centro Nacional de Excelencia Tecnológica en Salud, ed. Catálogo Maestro de Guías de Práctica Clínica. México D.F. 2016. ISBN: 9786077790624

32. Martínez Figueroa R, Martínez Figueroa C, Calvo Rodriguez R, Figueroa Poblete D. Osteoartritis (artrosis) de rodilla. Rev Chil Ortop y Traumatol. 2015;56(3):45-51. doi:10.1016/j.rchot.2015.10.005 ISSN: 07164548

33. Haywood C, Sumithran P. Treatment of obesity in older persons-A systematic review. Obes Rev. 2019;20(4):588-598. doi:10.1111/ obr.12815 PMID: 30645010

34. Concors SJ, Ecker BL, Maduka R, et al. Complications and Surveillance After Bariatric Surgery. Curr Treat Options Neurol. 2016;18(1):112. doi:10.1007/s11940-015-0383-0 PMID: 26860932

35. Quirante FP, Montorfano L, Rammohan R, et al. Is bariatric surgery safe in the elderly population? Surg Endosc. 2017;31(4):1538-1543. doi:10.1007/s00464-016-5050-3 PMID: 28039650

36. Koh CY, Inaba CS, Sujatha-Bhaskar S, Nguyen NT. Outcomes of laparoscopic bariatric surgery in the elderly population. Am Surg. 2018;84(10):1600-1603. PMID: 30747677

37. Bernstein A. Emerging patterns in overweight and obesity in Ecuador. Rev Panam Salud Publica/Pan Am J Public Heal. 2008;24(1):7174. doi:10.1590/S1020-49892008000700010

38. Vinan-Vega M, Diaz Vico T, Elli EF. Bariatric Surgery in the Elderly Patient: Safety and Short-time Outcome. A Case Match Analysis. Obes Surg. 2019;29(3):1007-1011. doi:10.1007/s11695-018-03633-2 PMID: 30536201

39. Buchwald H, Avidor Y, Braunwald E, et al. Bariatric surgery: A systematic review and meta-analysis. J Am Med Assoc. 2004;292(14):17241737. doi:10.1001/jama.292.14.1724 PMID: 15479938

40. Zambrano, A., Gaviria, A., Cobos-Navarrete, S. et al. The three-hybrid genetic composition of an Ecuadorian population using AlMs-InDels compared with autosomes, mitochondrial DNA and $Y$ chromosome data. Sci Rep 9, 9247 (2019). https://doi.org/10.1038/ s41598-019-45723-w 